\title{
МЕТОД ЭПСИЛОН-ПОДСТАНОВОК ДЛЯ ТЕОРИИ НАСЛЕДСТВЕННО КОНЕЧНЫХ МНОЖЕСТВ
}

\author{
(Представил Э. Тыугу)
}

\section{1. Введение}

Метод эпсилон-подстановок (ЭП) был введен Д. Гильбертом [ ${ }^{1}$ ] в рамках его программы обоснования математики с помощью теории доказательств и нашел широкое применение $\left[{ }^{2-6}\right]$. Исходный замысел Д. Гильберта состоял в исключении инфинитарных средств (использования связанных переменных, пробегающих бесконечную совокупность) из доказательств финитных утверждений - арифметических тождеств. Позднее метод ЭП был применен для извлечения числового содержания из арифметических доказательств, в частности, оценки сложности получаемых программ [3]. Существенно, что этот метод применим к традиционным (классическим) доказательствам и в отличие от многих других методов извлечения программ, нацеленных в основном на конструктивные системы, не требует модификации. Еще одно его важное преимущество перед генценовскими методами нечувствительность к пропозициональной структуре вывода: сложность извлекаемой программы (и скорость сходимости метода) измеряется только «глубиной вложенности» связанных переменных. При рассмотрении генценовских выводов для достижения того же эффекта приходится применять специальные приемы. Все это делает желательным обобщение метода ЭП с арифметики (для которой он был первоначально введен) на другие теории. Мы подробно рассмотрим теорию наследственно конечных множеств (НКМ, см., напр. $\left[{ }^{7}\right], \S 6$ гл. 1) и на примере нескольких ее формализаций продемонстрируем технику такого обобщения. Выбор именно этой теории обусловлен, в частности, использованием теории списков в теоретическом программировании (см., напр. $\left.\left[{ }^{8}\right]\right)$. Основной технический результат настоящей работы - сходимость метода ЭП для теории НКМ, которая доказана методом, введенным в $\left[{ }^{9}\right]$. Этот результат обобщает теорему Аккермана о сходимости метода ЭП для арифметики первого порядка (см. $\left.\left[{ }^{1}\right]\right)$. Большой вклад в фактическую реализацию предложенных автором соображений внес в своей дипломной работе А. Горбис; он является, по существу, соавтором настоящей статьи.

\section{2. Теория наследственно конечных множеств}

Напомним три эквивалентных (в смысле интерпретируемости) варианта этой теории в языке обычного классического исчислеңия прредикатов с отношениями $\in$, '=. 
Первый - просто теория множеств ZFC (система Цермело-Френкеля с аксиомой выбора см. $\left.\left[{ }^{7,10}\right]\right)$ без аксиомы бесконечности. Второй - (рассматривавшийся, например, А. Тарским) та же теория с отрицанием аксиомы бесконечности. Наконец, третий вариант (см. [11], начало раздела 2), который будет для нас основным и обозначается через HF - теория, содержащая константу $\varnothing$ (пустое множество), двухместную функцию * (добавление множеству одного элемента) и (в дополнение к исчислению предикатов с равенством) следующие аксиомы.

Аксиомы теории HF.

Объемность: $\forall z(z \in x \leftrightarrow z \in y) \rightarrow x=y$.

Пустое множество: $\sim y \in \varnothing$.

Добавление одного элемента: $u \in x * y \leftrightarrow u \in x \vee u=y$.

Индукция: $A(\varnothing) \& \forall x \forall y(A(x) \& A(y) \rightarrow A(x * y)) \rightarrow \forall z A(z)$.

Стандартная модель теории НF состоит из наследственно конечных множеств, которые получаются из $\varnothing$ с помощью операции $*$. Напомним для дальнейшего, что натуральные числа в этой модели определяются как множества $\varnothing$ (число 0$), \varnothing * \varnothing$ (т. е. $\{\varnothing\}$, число 1$)$, $1 * 1$ (т. е. $\{0,1\}$, число 2) и т. д. При этом натуральное число $n=$ $=\{0,1, \ldots, n-1\}$ имеет, как множество, мощность $n$, т. е. содержит ровно $n$ элементов.

Другая важная модель теории HF введена В. Аккерманом. Она использует взаимно однозначную нумерацию $n(x)$ наследственно конечных множеств $x$ натуральными числами, при которой элементы множества кодируются разрядами двоичного разложения

$$
n(\{a, b, \ldots, c\})=2^{n(a)}+2^{n(b)}+\ldots+2^{n(c)} ; \quad n(\varnothing)=0 .
$$

Чисто арифметическое определение получается, если ввести арифметический предикат $\in^{\prime}$ соотношением $k \in \in^{\prime} m \leftrightarrow\left(\left[m / 2^{k}\right]\right.$ нечетно). Следующее утверждение об эквивалентности рассмотренных формулировок и арифметики первого порядка хорошо известно (см., напр., $\left[{ }^{12}\right]$ ) и легко проверяется непосредственно.

Т е о р м а 1. В модели Аккермана выполнены (и даже доказуемы в арифметике первого порядка) все аксиомы ZFC, кроме аксиомы бесконечности (которая опровергается). После обратного перевода все арифметические аксиомь выводимы в ZFC без аксиомы бесконечности и в $\mathrm{HF}$.

В дальнейшем используется монотонность кодировки Аккермана (1) относительно принадлежности элемента множеству и включения множеств:

$$
x \in y \rightarrow n(x)<n(y), \quad x \subset y \& x \neq y \rightarrow n(x)<n(y) .
$$

\section{3. Язык с эпсилон-символом}

Первый шаг гильбертовского метода ЭП $\left[{ }^{1}\right]$ - переход к эквивалентной бескванторной формальной системе с т. н. эпсилон-символом. Кванторы исключаются из языка, куда добавляется новое правило образования термов: если $A-$ формула, то $\varepsilon x A-$ терм (который читается «объект $x$, удовлетворяющий условию $A »)$. Вхождения переменной $x$ в этот терм - связанные. Вместо постулатов для кванторов используются логические эпсилон-аксиомы

$$
A_{x}[t] \rightarrow A_{x}[\varepsilon x A],
$$

где $A_{x}[t]$ или, короче, $A[t]$ - результат подстановки терма $t$ вместо всех свободных вхождений переменной $x$ в $A$ (с обычными предо- 
сторожностями, включая переименование связанных переменных во избежание коллизий). Кроме того, сохраняется правило подстановки терма вместо свободной предметной переменной $A / A[t]$ или, эквивалентным образом, все аксиомы понимаются как схемы аксиом. Так как некоторые из кванторных аксиом описывают вычислимые функции, для этих функций вводятся явные символы. В случае арифметики $\left[{ }^{1}\right]$ - это сложение, умножение и вычитание 1 (предшествующее натуральное число). У нас используются функция $d(x, y)$, дающая элемент симметрической разности $x \Delta y$, если она непуста

$$
x \neq y \rightarrow \sim(d(x, y) \in x \leftrightarrow d(x, y) \in y),
$$

и функция $p(x, y)$, дающая множество $x-\{y\}$,

$$
z \in p(x, y) \leftrightarrow z \in x \& z \neq y .
$$

Наконец, вместо аксиом индукции вводятся математические эnсилон-аксиомы, выражающие, что $\varepsilon x A$ в некотором смысле минимален. В нашем случае требуется минимальность по принадлежности и по включению

$$
y \in \varepsilon x A \rightarrow \sim A_{x}[y], \quad y \in \varepsilon x A \rightarrow \sim A_{x}[p(\varepsilon x A, y)] .
$$

Обозпачим через HFe теорию в языке с эпсилон-символом, аксиомами которой служат аксиомы исчисления высказываний с равенством, экстенсиональность в форме (4), аксиомы (5), аксиомы пустого множества и добавления, а также логические и математические эпсилон-аксиомы (3), (6) (понимаемые как схемы аксиом). Мы говорим, что (3), (6) - аксиомы для эпсилон-терма $є x A$. Следующий стандартный шаг метода ӘП - погружение кванторной теории в ее эпсилонвариант.

Т е о р м а 2. При переводе

$$
\exists x A \mapsto A_{x}[\varepsilon x A] ; \quad \forall x A \mapsto A_{x}[\varepsilon x \sim A]
$$

все аксиомы и правила системы НF становятся выводимыми в $\mathrm{HFe.}$

Д ок аз а тельст во. Аксиома $A[t] \rightarrow \exists x A$ переходит в логическую эпсилон-аксиому, кванторное правило $A / \forall x A$ переходит в применение правила подстановки $A / A[\varepsilon x \sim A]$, которое выводимо. Бескванторные аксиомы сохраняются. Аксиома экстенсиональности системы HF переходит в импликацию $(Z \in x \leftrightarrow Z \in y) \rightarrow x=y$, где $Z$ есть $\varepsilon z \sim(z \in x \leftrightarrow z \in y)$. Она получается из логической эпсилон-аксиомы для $Z$ и аксиомы экстенсиональности системы HF

$$
(Z \in x \leftrightarrow Z \in y) \rightarrow(d(x, y) \in x \leftrightarrow d(x, y) \in y) \rightarrow x=y .
$$

Наконец, рассмотрим перевод НF-аксиомы индукции

$$
B[\varnothing] \&(B[X] \& B[Y] \rightarrow B[X * Y]) \rightarrow B[Z],
$$

где $\quad Z=\varepsilon z \sim B[z], \quad Y=\varepsilon y \sim(B[x] \& B(y) \rightarrow B(x * y))$, $X=\varepsilon x \sim(B[x] \& B[Y] \rightarrow B[x * Y])$.

Допустим, что верна посылка импликации (8), и докажем $B[Z]$. При $Z=\varnothing$ это следует из $B[\varnothing]$. Поэтому считаем, что $Z \neq \varnothing$. Из (4) следует, что $d(Z, \varnothing) \in Z$. Вводя обозначения $y=d(Z, \varnothing), x=p(Z, y)$, получаем $Z=x * y$, так как в силу (5)

$$
u \in v \rightarrow v=p(v, u) * v .
$$

В силу математических $\varepsilon$-аксиом (6) для $Z$ имеем $\sim \sim B[x]$ и $\sim \sim B[y]$, т. е. $B[x] \& B[y]$. В силу логических $\varepsilon$-аксиом для $X, Y$ имеем из посылки (8) $B[x] \& B[y] \rightarrow B[x * y]$, откуда $B[x * y]$, т, е. $B[Z]$, что и требовалось. 


\section{4. Описание метода ЭП}

Зафиксируем какую-нибудь нумерацию $n(x)$ наследственно конеч́ных множеств $x$, удовлетворяющую условиям (2). Описываемый ниже аналог метода Гильберта, который мы назовем методом ЭП (эпсилонподстановок) для теории НКМ (наследственно конечных множеств), состоит, по существу, в том, что значение терма вхA берется равным $\mu x A$ - наименьшему в смысле нумерации $n$ множеству $x$, удовлетворяющему условию $A$.

Применяются две меры сложности термов: степень и ранг. Степень терма - это обычная мера гнездности $\varepsilon$-символов: $\operatorname{deg}(t)=0$, если $t$ не содержит $\varepsilon ; \operatorname{deg}\left(f\left(t_{1}, \ldots, t_{n}\right)\right)=\max \left(\operatorname{deg}\left(t_{1}\right), \ldots, \operatorname{deg}\left(t_{n}\right)\right)$; $\operatorname{deg}(\varepsilon x A)=1+\max (\operatorname{deg}(t): t$ отличен от $\varepsilon x A$ и входит в $\varepsilon x A$ свободно $)$ Ранг терма - мера гнездности по связанным вхождениям: $\operatorname{rk}(t)=0$, если $t$ не содержит $\varepsilon ; \operatorname{rk}\left(f\left(t_{1}, \ldots, t_{n}\right)\right)=\max \left(\operatorname{rk}\left(t_{1}\right), \ldots, \operatorname{rk}\left(t_{n}\right)\right)$; $\operatorname{rk}(\varepsilon x A)=1+\max (\operatorname{rk}(t): t$ имеет в $A$ вхождение, содержащее $x$ свободно).

П р и м е р: $\quad t=\varepsilon x(\varepsilon y(x * y=\varepsilon z(z=5)))=1, \quad \operatorname{deg}(t)=2, \quad \operatorname{rk}(t)=2$. Результат замены в эпсилон-терме $t$ отличных от $t$ максимальных свободных вхождений подтермов на переменные называется его эпсилон-матрицей. В нашем примере эпсилон-матрицей терма будет $\varepsilon x(\varepsilon y(x * y=a)=b)$. Ранг эпсилон-терма равен рангу его эпсилонматрицы. Степень любой эпсилон-матрицы равна 1.

Пусть $\mathfrak{R}$ - некоторая система (конечный набор) $\varepsilon$-матриц и $S$ - функция, сопоставляющая каждой эпсилон-матрице $M=$ $=\varepsilon x A\left(x, a_{1}, \ldots, a_{l}\right)$ из $\mathfrak{M}$ некоторую финитную $l$-местную функцию на НKM, т. е. со значениями в НKM, отличную от $\varnothing$ лишь на конечном множестве значений аргументов. Функцию $S$ можно распространить на любые постоянные (т. е. не содержащие переменных) термы и формулы, содержащие только эпсилон-термы с матрицами из $\mathfrak{M}$. Множество $S(t)$ определяется индукцией по построению терма $t$ с индуктивным переходом $S\left(\varepsilon x A\left(x, t_{1}, \ldots, t_{l}\right)\right)=S(M)\left(S\left(t_{1}\right), \ldots, S\left(t_{l}\right)\right)$, где $M=\varepsilon x A\left(x, a_{1}, \ldots, a_{l}\right), S\left(f\left(t_{1}, \ldots, t_{l}\right)\right)=f\left(S\left(t_{1}\right), \ldots, S\left(t_{l}\right)\right)$. Истинностное значение $(0,1)$ формулы $F$ определяется в соответствии с обычными булевскими правилами индукцией по построению $F$ с базисом $S(t=r)=1$, если $S(t)=S(r) ; S(t \in r)=1$, если $S(t) \in S(r)$. Функцию $S$ будем называть ЭП (эпсилон-подстановкой) для данной системы $\varepsilon$-матриц, если для каждой матрицы $M=\varepsilon x A\left(x, a_{1}, \ldots, a_{k}\right)$ из этой системы и каждого терма $t=\varepsilon x A\left(x, n_{1}, \ldots, n_{k}\right)$ из неравенства $S(t) \neq \varnothing$ следует, что $S(t)$ есть наименьшее в смысле нумерации $n$ множество $m$, такое что $S\left(A\left(m, n_{1}, \ldots, n_{k}\right)\right)=1$.

Предложенный Д. Гильбертом метод исключения трансфинитного (в рассматриваемом случае - эпсилон-символа) из доказательств финитных формул состоял в построении выполняющей ЭП для любого конечного набора постоянных эпсилон-аксиом, т. е. такой постановки $S$, что $S(E)^{\prime}=1$ для любой формулы $E$ из рассматриваемого набора. Действительно, можно считать, что данное доказательство $d$ не содержит свободных переменных (их можно заменить на $\varnothing$ ) и содержит только такие эпсилон-термы, матрицы которых участвуют в используемых эпсилон-аксиомах (остальные эпсилон-термы можно заменить на Ø). Применение выполняющей подстановки $S$ ко всем эпсилонтермам из $d$ не меняет последней формулы вывода, устраняет эпсилонтермы и переводит все формулы в доказуемые.

Если теперь дано доказательство $d$ формулы $F$, не содержащей переменных, то применим подстановку $S_{d}$, выполняющую все эпсилонаксиомы $E$, входящие в $d$. Сама формула $F$ при этом не изменится, и мы получим ее доказательство, не содержащее эпсилон-символа, что и требуется. 
Для построения выполняющей подстановки применим следующий метод последовательных приближений. Фиксируется конечный набор $\mathcal{E}$ постоянных эпсилон-аксиом (3), (6), и список эпсилон-матриц всех эпсилон-термов из этого набора обозначается через $\mathfrak{M}$. Считается, что $\mathfrak{M}$ составлен в порядке возрастания рангов термов $\varepsilon x A$ : сначала матрицы ранга 1, затем - ранга 2 и т. д.; считается, что формулы (3) выписаны в том же порядке. Исходная подстановка $S_{0}$ - нулевая: $S(M)=\varnothing$ (нулевая функция) для любой матрицы $M$. Если подстановка $S$ уже определена и она не является выполняющей для $\boldsymbol{E}$, то выбирается первая из рассматриваемых эпсилон-аксиом $E$, для которой $S(E)=0$. Пусть $E$ - логическая эпсилон-аксиома. С использованием соответствующей эпсилон-матрицы $\varepsilon x A\left(x, a_{1}, \ldots, a_{n}\right)$ она записывается в виде $A\left(t, t_{1}, \ldots, t_{n}\right) \rightarrow A\left(\varepsilon x A\left(x, t_{1}, \ldots, t_{n}\right), t_{1}, \ldots, t_{n}\right)$. Если $u^{*}$ обозначает $S(u)$, то из $S(E)=0$ получаются $S\left(A\left(t^{*}, t_{1}{ }^{*}\right)\right)=1$ и $S\left(A\left(\left(\varepsilon x A\left(x, t_{1}^{*}\right)^{*}, t_{1}^{*}\right)=0 \quad\right.\right.$ (для простоты записи считается, что $\left.n=1\right)$. В следующей $\varepsilon$-подстановке $S^{\prime}$ сохраняются те же значения, что и в $S$ для всех матриц, имеющих не больший ранг, чем рассматриваемая, «сбрасываются» на $\varnothing$ все значения матриц, имеющих больший ранг, и добавляется к оценке $S(M)$ для рассматриваемой матрицы $M$ значение $S^{\prime}(M)\left(t_{1}^{*}\right)=m$, где $m-$ наименьшее, не превосходящее $t^{*}$ HKM такое, что $S\left(A\left(m, t_{1}^{*}\right)\right)=1$. Этим завершается определение $S^{\prime}$. Теперь $S_{i+1}=S_{i}{ }^{\prime}$ для $i \geqslant 0$. Математические эпсилон-аксиомы (6) рассматриваются аналогично.

\section{5. Существование выполняющей ЭП}

Все исследования ЭП в той или иной мере используют непрерывность рассматриваемых операций. Напомним соответствующие свойства, которые понадобятся при доказательстве сходимости метода ЭП.

Заметим, что этот метод можно применять к системам эпсилонаксиом, содержащих символы произвольных функций, отображающих НКМ в НКМ.

Рассмотрим систему эпсилон-аксиом, содержащих, возможно, (свободные) переменные $\bar{f}$ для функций. Запишем такую систему в виде $\mathcal{E}(\bar{f})$. Применение метода эпсилон-подстановок возможно только после того, как вместо переменных $\bar{f}$ будут подставлены символы конкретных функций $\bar{\varphi}$, что даст систему $\mathcal{E}(\bar{\varphi})$.

Т еорем а 3. (а) Для любой формулы $A(\bar{f})$, не содержащцей индивидных переменных, для любой системы $\bar{\varphi} с$ подходящим числом аргументов найдется такое конечное семейство Ф НКМ, что для любых функций $\bar{\psi}$, совпадающих $с \bar{\varphi}$ на всех аргументах из Ф, верно $A(\bar{\varphi}) \leftrightarrow A(\bar{\psi})$.

(b) Для каждой системы $\varepsilon(\bar{f})$ эпсилон-аксиом со свободными переменными $\bar{f}$, для любой системы функций $\bar{\varphi}$ с подходящим числом аргументов и любой эпсилон-подстановки $S$ для системы $\&(\bar{\varphi})$ найдется такое конечное семейство ФФ НКМ, что для любых функций $\bar{\psi}$, совпадающих с $\bar{\varphi}$ на всех аргументах из Ф

(b1) $S(A(\bar{\varphi}))=S(A(\bar{\psi}))$ для любой эпсилон-аксиомы $A$ из системы है;

(b2) подстановка $S^{\prime}$, следующая за $S$, для системы $\mathcal{E}(\bar{\psi})$ та же, что $u$ для $\varepsilon(\bar{\varphi})$. 
Да оказ а тельство. При вычислении истинностного значения $A(\bar{\varphi})$ и построении следующей за $S$ ЭП $S^{\prime}$ вычисляются значения различных функций при различных значениях аргументов. Ф - совокупность всех таких значений аргументов.

Если интересоваться только существованием выполняющей подстановки (а не сходимостью метода), то результат можно получить сравнительно просто. Приводимое ниже рассуждение, по-видимому, известно специалистам, но автор не нашел его в литературе.

Т еорем а 4. Для любой конечной системь \& эпсилон-аксиом (3), (6) существует выполняющая ЭП S, являющая «срезкой» оператора минимизации: для постоянных термов єхF имеет место

$$
S(\varepsilon x F) \neq \varnothing \rightarrow S(\varepsilon x F)=\mu x(S(F)=1),
$$

где $\mu x F$ означает наименьшее в смысле нумерации $n$ HКM $x, y \partial о в-$ летворяющее условию $F, u$ эпсилон-символы внутри $F$ понимаются так же.

Док а 3 а тельст во. Подготавливая применение теоремы $3(a)$, выпишем все встречающиеся в $\mathcal{E}$ различные эпсилон-матрицы

$$
\varepsilon x_{1} F_{1}\left(x_{1}, \mathbf{y}_{1}\right), \ldots, \varepsilon x_{n} F_{n}\left(x_{n}, \mathbf{y}_{n}\right)
$$

или, короче,

$$
\varepsilon_{1}\left(\mathbf{y}_{1}\right), \ldots, \varepsilon_{n}\left(\mathbf{y}_{n}\right) .
$$

Заменяя в $\varepsilon$ матрицы $\varepsilon_{1}, \ldots, \varepsilon_{n}$ на новые свободные функциональные переменные $f_{1}, \ldots, f_{n}$ с соответствующим числом аргументов и соединяя все формулы знаком конъюнкции, получаем бескванторную формулу $A(\mathbf{f})=A\left(f_{1}, \ldots, f_{n}\right)$ такую, что $\& \mathcal{E}=A\left(\varepsilon_{1}, \ldots, \varepsilon_{n}\right)$. Заметим, что подстановка $\varphi_{i}=\mu x_{i} F_{i}\left(x_{i}, y_{i}\right)$ вместо $\varepsilon_{i}$ делает все эпсилонаксиомы истинными. (Именно рассмотрение этих $\mu$-операторов - основной источник неконструктивности нашего доказательства.) Таким образом, формула $A\left(\varphi_{1}, \ldots, \varphi_{n}\right)$ истинна. Остается только применить теорему $3(a)$.

\section{6. Сходимость метода ЭП}

Наша цель - доказательство следующего утверждения.

Т е о е м а 5. Для любой конечной системы эпсилон-формул (2) метод ЭП сходится, т. е. найдется $m$ такое, что $S_{m}-$ выполняющая подстановка.

Приведенное ниже доказательство - обобщение рассуждения из $\left[{ }^{9}\right]$. Внимательный читатель заметит сходство с доказательством теоремы 4.

Будем говорить, что ЭП $S_{1}$ продолжает ЭП $S$ (обозначение $\left.S_{1} \geqslant S\right)$, если все ненулевые значения из $S$ сохраняются в $S_{1}$.

Следующее утверждение обобщает известные результаты Дж. фон Неймана и В. Аккермана (см. $\left[{ }^{1}\right]$, с. 107-111).

Т еорем а 6 (а). Метод ЭП сходится для эпсилон-формул ранга 1. (б). Более того, любая последовательность продолжающих друг друга ЭП $S_{1}, S_{2}, \ldots$ такая, что при каждом $k S_{k+1}(t) \neq S_{k}(t)$ для некоторого терма $t$, входящего в данную систему $\varepsilon$ в-формул ранга 1 , содержит не более $2^{m}$ членов, где $m$ - количество в-термов в $\varepsilon$. В частности, метод ЭП сходится за $2^{m}$ шагов.

(в) Если $\varepsilon-$ - система эпсилон-формул ранга $1, S^{+} \geqslant S-Э П u$ $S^{+}(t) \neq S(t)$ для некоторого терма $t$, входящего в $\mathcal{E}$, то найдется эпсилон-терма $r$, входящий в $t$ и такой, что $S(r)=\varnothing$ и $S^{+}(r) \neq \varnothing$. 
Даказ а тельство. (a) следует из (б). Чтобы вывести (б) из $(в)$, составим список $t_{1}, \ldots, t_{m}$ из $\varepsilon$-термов, входящих в $\mathcal{E}$, в порядке возрастания (точнее, неубывания) степеней и положим

$$
i_{S}=2^{m-1} \delta_{1}+2^{m-2} \delta_{2}+\ldots+2 \delta_{m-1}+\delta_{m},
$$

где $\delta_{i}=\operatorname{sgn}\left(S\left(t_{i}\right)\right)$. Имеем $i_{S}<2^{m}$. Далее, если $S_{k+1}$ и $S_{k}$ связаны так, как сказано в пункте (б), получим, в силу пункта $(8), \quad i_{S_{k+1}}>i_{S_{k}}$ что и доказывает (б).

Осталось доказать (в). Применим индукцию по степени терма $t$. В базисе индукции $\operatorname{deg}(t)=1$, поэтому из $S^{+}(t) \neq S(t)$ следует $t=\varepsilon x A\left(x, r_{1}, \ldots, r_{k}\right)$, причем $S(t)=\varphi\left(n_{1}, \ldots, n_{k}\right), S^{+}(t)=\varphi^{+}\left(n_{1}, \ldots, n_{k}\right)$, где $n_{1}, \ldots, n_{h}-$ значения термов $r_{1}, \ldots, r_{k}$ (они не содержат $s$ ), а $\varphi, \varphi^{+}-$функции, сопоставленные $\varepsilon$-матрице $\varepsilon x A\left(x, a_{1}, \ldots, a_{k}\right)$ в $S, S^{+}$. Допустим, что $\varphi\left(n_{1}, \ldots, n_{k}\right)=S(t) \neq \varnothing$. Тогда ввиду $S^{+} \geqslant S$ имеем $\varphi^{+}\left(n_{1}, \ldots, n_{k}\right)=\varphi\left(n_{1}, \ldots, n_{k}\right)$, т. е. $S(t)=S^{+}(t)$ вопреки предположению. Значит, $S(t)=\varnothing$, что вместе с $S^{+}(t) \neq S(t)$ дает $S^{+}(t) \neq \varnothing$.

Инду к ти вны й пе реход. В случае, когда $t=f\left(t_{1}, \ldots, t_{k}\right)$, результат сразу получается из индуктивного предположения, так как должно быть $S\left(t_{i}\right) \neq S^{+}\left(t_{i}\right)$ для некоторого $i \leqslant k$. Остался случай $t=\varepsilon x A(x, u)$, где $\varepsilon x A(x, a)$ - матрица терма $t$ (для простоты записи предполагаем, что у этой матрицы всего один аргумент). Имеем $S(t)=\varphi(S(u)), S^{+}(t)=\varphi^{+}\left(S^{+}(u)\right)$, где $\varphi, \varphi^{+}-$функции, сопоставленные матрице $\varepsilon x A(x, a)$ при подстановках $S, S^{+}$. Если $S(u) \neq S^{+}(u)$, то применимо индуктивное предположение. Поэтому будем считать, что $S(u)=S^{+}(u)$, и обозначим это число через $u^{*}$. Из $\varphi\left(u^{*}\right)=S(t) \neq S^{+}(t)=$ $=\varphi^{+}\left(u^{*}\right)$ следует, как и в базисе индукции $\varphi\left(u^{*}\right)=\varnothing, \varphi^{+}\left(u^{*}\right) \neq \varnothing$, т. е. можно взять $r=t$, что и требовалось доказать.

Т еорем а 7. При переходе от $S \kappa$ следующей подстановке $S^{\prime}$ сохраняются все ненулевые значения в-матриц минимального имеющегося ранга.

Предложение непосредственно следует из определения $S^{\prime}$.

Докажем теорему 5 индукцией по рангу данной системы $\varepsilon$-формул. Базисом служит наша теорема 6. Предположим, что для данного $r$ теорема доказана, и рассмотрим систему $\mathcal{E}$ ранга $r+1$. Введем вспомогательную систему $\mathcal{E}^{+}(\bar{f})$, полученную из $\mathcal{E}$ заменой всех эпсилонматриц минимального ранга (считаем, что он равен 1) на функциональные символы $\bar{f}$ с соответствующим числом мест и вычеркиванием эпсилон-аксиом минимального ранга. Рассмотрим также систему $\mathcal{E}^{1}(\bar{g})$, полученную из $\mathcal{E}$ заменой всех матриц $M$ ранга больше 1 на функциональные символы $\bar{g}$ с соответствующим числом мест и вычеркиванием соответствующих им эпсилон-аксиом.

Допустим теперь (для приведения к противоречию), что метод ЭП для системы $\varepsilon$ не сходится, т. е. ни одна из подстановок $S_{0}, S_{1}, S_{2}, \ldots$ не является выполняющей для $\mathcal{E}$. Каждую ЭП $S_{i}$ для $\mathcal{E}$ можно представить в виде $\left(\bar{\varphi}_{i}, \bar{\psi}_{i}\right)$, где $\varphi_{i}-$ подстановки для $\varepsilon$-матриц ранга 1 , $\bar{\psi}_{i}$ - подстановки для остальных $\varepsilon$-матриц. При этом в силу теоремы 6 и того, что ранг системы $\mathcal{E}^{1}$ равен 1, можем утверждать, что функции $\bar{\varphi}_{i+1}$ являются продолжениями функций $\bar{\varphi}_{i}$, т. е. из $\bar{\varphi}_{i}(\bar{x}) \neq \varnothing$ следует $\bar{\varphi}_{i+1}(\bar{x})=\bar{\varphi}_{i}(\bar{x})$. Пусть $\bar{\varphi}-$ объединение всех $\bar{\varphi}_{i}$, т. е. для любой функции $\theta$ из списка $\bar{\varphi}$ значение $\theta(\bar{x})$ равно $z$, если для некоторого $i$ верно $\theta_{i}(\bar{x})=z \neq \varnothing$; в противном случае $\theta(\bar{x})=\varnothing$. Применяя индуктивное предположение к системе $\mathcal{E}^{+}(\bar{\varphi})$ ранга $r$, найдем номер $h$, при котором подстановка $S_{h}^{\bar{\varphi}}$ для системы $\mathcal{E}^{+}(\bar{\varphi})$ является выполняющей. Ис- 
пільзуя теорему 3 (в2), найдем конечное семейство $\Phi+$ НКМ для $\mathcal{E}^{+}(\overline{\bar{\varphi}})$ и всех подстановок $S_{0}^{\bar{\varphi}}, \ldots, S_{h}^{\bar{\varphi}}$. Затем найдем номер $i_{0}$, начиная с которого все ненулевые значения функций $\bar{\varphi}$ для аргументов из $\Phi^{+}$ равны значению $\bar{\varphi}_{i_{0}}$.

Рассмотрим подробнее последовательность $\left(\bar{\varphi}_{i}, \bar{\psi}_{i}\right)$ для $i \geqslant i_{0}$. Возможны два случая.

ф-переход: $\bar{\varphi}_{i+1}=\left(\bar{\varphi}_{i}\right)^{\prime}, \bar{\psi}_{i+1}=\varnothing . \bar{\varphi}_{i}$ не является выполняющей подсста новкой для $\mathcal{E}^{1}\left(\bar{\psi}_{i}\right)$.

$\psi$-переход: $\bar{\varphi}_{i+1}=\bar{\varphi}_{i} ; \bar{\psi}_{i+1}=\left(\bar{\psi}_{i}\right)^{\prime} \cdot \bar{\varphi}_{i}-$ выполняющая подстановка для $\mathcal{E}^{1}\left(\bar{\psi}_{i}\right)$

Пусть $h_{1}=2^{m_{1}}$, где $m_{1}-$ количество $\varepsilon$-термов в системе $\varepsilon^{1}(\bar{g})$. По теореме 4 любая последовательность соседних $\varphi$-переходов содержит не более $h_{1}$ членов. В силу выбора $\Phi^{+}, i_{0}$ любая начинающаяся с $\varnothing$ последовательность соседних $\psi$-переходов $\bar{\psi}_{k}=\varnothing, \bar{\psi}_{k+1}, \ldots$ совпадает с последовательностью $\Psi=S_{0}^{\bar{\Phi}}, \ldots, S_{h}^{\bar{\varphi}}$, и потому она содержит ровно $h$ членов. Таким образом, последовательность $\left(\bar{\varphi}_{i}, \bar{\psi}_{i}\right)$ для $i>i_{0}$ имеет вид

$$
\Phi_{1} ; \Psi ; \Phi_{2} ; \Psi ; \ldots,
$$

где $\Phi_{i}$ и $\Psi$ - участки, состоящие из последовательных $\varphi$ - и $\psi$-переходов соответственно. Считая для простоты записи, что $i_{0}=0$, мы можем представить участок $\Psi ; \Phi_{i+1}$ в следующем виде:

$\left.\left.\left(\bar{\varphi}^{i}, \bar{\psi}_{0}\right), \quad\left(\bar{\varphi}^{i}, \bar{\psi}_{1}\right), \ldots, \quad\left(\bar{\varphi}^{i}, \bar{\psi}_{h}\right) ;\left(\overline{(\varphi}^{i}\right)^{\prime}, \varnothing\right),\left(\overline{(\varphi}^{i}\right)^{\prime \prime}, \varnothing\right), \ldots,\left(\left(\bar{\varphi}^{i}\right)^{\left(l_{t}\right)}, \varnothing\right)$,

где $\bar{\varphi}_{i}-$ последняя $\varepsilon$-подстановка участка $\Phi_{i}$.

Длина $1_{i}$ участка $\Phi_{i+1}$ может быть равна единице, т. е. может быть $\bar{\varphi}^{i+1}=\left(\overline{\varphi^{i}}\right)^{\prime}$. Ни при каком $i$ подстановка $\bar{\varphi}^{i}$ не является выполняющей для $\mathcal{E}^{1}\left(\bar{\psi}_{h}\right)$, иначе $\varphi$-переход после участка $\psi$ был бы невозможен. Это означает, что при каждом из рассмотренных значений $i$ верно $\left(\bar{\varphi}^{i}\right)^{\prime}(t) \neq\left(\overline{\varphi^{i}}\right)(t)$ для некоторого терма $t$, входящего в $\mathcal{E}^{1}\left(\bar{\psi}_{h}\right)$. Поскольку последовательные $\varphi$-компоненты - продолжения друг друга, то в силу части 6 теоремы 6 , примененной к $\mathcal{E}^{1}\left(\bar{\psi}_{h}\right)$, получаем, что количество $\varphi$-участков не превосходит $h_{1}$, т. е. поеледовательность ЭП обрывается, что и требовалось доказать.

\section{7. Распространение на другие формулировки теории НКМ}

Теорема 7 дает метод построения выполняющей ЭП для системы HFe. Проиллюстрируем обобщение этого метода и покажем, как получить выполняющую подстановку по выводу в ZFC без аксиомы бесконечности.

Напомним формулировки аксиом этой системы.

Аксиома объемности: $\forall z(z \in x \leftrightarrow z \in y) \rightarrow x=y$.

Аксиома выделения: $\exists c \forall x(x \in c \leftrightarrow(x \in a \& F(x)))$.

Аксиома объединения: $\exists u \forall x(x \in u \leftrightarrow(\exists z \in y) x \in z)$.

Аксиома степени: $\exists p \forall x(x \in p \leftrightarrow y \subset x)$.

Аксиома подстановки: $\forall x y z(F(x, y) \& F(x, z) \rightarrow y=z) \rightarrow$

Аксиома регулярности $\rightarrow \exists s \forall y(y \in s \leftrightarrow(\exists x \in u) F(x, y))$

Аксиома выбора: $\exists c(\forall y \subset x)(y \neq \varnothing \rightarrow(\exists ! z \in y)\langle y, z\rangle \in c)$. 
Оิпишем два способа получения ӘП, реализующих эти аксиомы. Под реализацией формулы $\exists x A(x)$, начинающейся с квантора существования, понимается НҚМ $m$, такое, что выполнено $A(m)$. При этом следует помнить, что если формула $A$ содержит кванторы, то выполнение $A(m)$ понимается в методе ЭП нестандартно: ведь области значений связанных переменных ограничиваются конечными семействами.

\section{1) Простейший и универсальный способ}

Каждая из рассматриваемых аксиом выводима в системе HF. Применяя теорему 2, получаем вывод в системе HFe. При этом формула $\exists x A(x)$ перейдет в $A^{*}\left(\varepsilon x A^{*}\right)$, где $A^{*}-$ перевод формулы $A$. Применяя к полученному выводу метод ЭП, получаем подстановку для $\varepsilon x A^{*}$, которая и является искомой.

\section{2) Введение функций, реализующих аксиомы}

Этот способ позволяет выполнить некоторые экзистенциальные утверждения стандартно, а не приблизительно, как в методе ЭП. Предположим, что имеется формула $B(x)$ не содержащая связанных переменных и такая, что $A(x) \leftrightarrow B(x)$. Тогда выполнение $\exists x B(x)$ всегда стандартно, а имея $B(m)$, мы получаем и $A(m)$. Иногда проще построить $B(x)$ такую, что $B(x) \rightarrow A(x)$ и $\exists x B(x)$. Формулу $B(x)$ чаще всего получают, устраняя из $A(x)$ кванторы с помощью (скулемовских) функций. Иногда это позволяет заменить существовательную аксиому на бескванторную так, что она вообще выпадает из рассмотрения в методе ЭП. При этом существенно, чтобы все вводимые функции были вычислимы (на НКМ). Один пример такого преобразования у нас уже был: при переходе от $\mathrm{HF}$ к HFe в разделе 2 мы ввели функцию $d$, выбирающую элемент в симметрической разности двух множеств, если она непуста. Это позволило скулемизировать аксиому объемности. Аксиома выбора следует из соотношения $y \neq \varnothing \rightarrow$ $\rightarrow d(y, \varnothing) \in y:$ в качестве функции выбора на множестве $x$ можно взять $\{\langle y, d(y, \varnothing)\rangle: y \subset x \& y=\varnothing\}$.

Заметим, что при переводе в эпсилон-язык можно не исключать ограниченные кванторы (вида $(\exists y \in t))$.

Действительно, все наши рассуждения, связанные с методом ЭП, проходят без изменений, если формулы с ограниченными кванторами (короче, ограниченные формулы) трактовать как бескванторные. При этом выполнение формулы $\exists x A(x)$ с ограниченной $A$ будет стандартным.

Так обстоит дело с аксиомами объединения, степени, регулярности. Учитывая, что соответствующие скулемовские функции вычислимы на НKM, мы можем переписать эти аксиомы в виде:

$$
\begin{gathered}
x \in U(y) \leftrightarrow(\exists z \in y) x \in z, \quad x \in P(y) \leftrightarrow(\forall z \in x) z \in y, \\
x \neq \varnothing \rightarrow(\operatorname{reg}(x) \in x \&(z \in \operatorname{reg}(x) \rightarrow z \notin x)) .
\end{gathered}
$$

С другой стороны, не видно, как можно было бы добиться стандартного выполнения аксиом выделения и подстановки.

Как и следовало ожидать, нельзя просто включить в рассмотрение аксиому бесконечности

$$
\exists \omega\left(\varnothing \in \omega \&(\forall x \in \omega) x^{\prime} \in \omega\right),
$$

где $x^{\prime}$ обозначает $x \cup\{x\}$.

Эту формулу можно выполнить при любой конкретной ЭП вместо подчиненного эпсилон-терма

$$
X(\omega)=\varepsilon x\left(x \in \omega \& x^{\prime} \notin \omega\right) .
$$


Действительно, вводя обозначение $\operatorname{Prog}(f, z)=\varnothing \in z \&(f(z) \in z \rightarrow$ $\left.\rightarrow(f(z))^{\prime} \in z\right)$, мы видим, что для любой финитной функции $S(\omega)$ можно найти $\mathrm{HKM} \Omega$ такое, что верно $\operatorname{Prog}(S, \Omega)$, т. е.

$$
\varnothing \in \Omega \&\left(S(\Omega) \in \Omega \rightarrow\left((S(\Omega))^{\prime} \in \Omega\right)\right) .
$$

Для этого берем натуральное положительное число $n$ (т. е. НКМ вида $\left.\left\{\varnothing, \varnothing^{\prime}, \varnothing^{\prime \prime}, \ldots, \varnothing(n-1)\right\}\right)$, удовлетворяющее условию $S(n) \neq$ $\neq n-1$. Такое число найдется, так как $\mathrm{S}(n)=\varnothing$, начиная с некоторого места. Условие (11) выполнено для $\Omega=n$, так как из $S(\Omega) \in \Omega$, $S(\Omega) \neq \Omega-1$ следует, что $(S(\Omega))^{\prime} \in \Omega$.

Тем не менее, наше доказательство существования выполняющей ЭП (теорема 5) не проходит, так как дополнительная эпсилон-аксиома имеет вид

$$
\operatorname{Prog}(X, \Omega) \text {, }
$$

где

$$
\Omega=\varepsilon \omega\left(\varnothing \in \omega \&\left(X(\omega) \in \omega \rightarrow(X(\omega))^{\prime} \in \omega\right)\right),
$$

но ее нельзя выполнить (при понимании $X(\omega)$ как $\mu x\left(x \in \omega \rightarrow x^{\prime} \in \omega\right)$ ) никаким наследственно-конечным множеством в отличие от эпсилонаксиом (3); (6) в разделе 3 , которые выполняются $\mu$-символом. По той же причине не проходит и доказательство сходимости метода ЭП (теорема 7), так как в ней используется возможность выполнить все эпсилон-аксиомы при подстановке любой (а не только финитной) функции $\bar{\varphi}$ вместо функциональной переменной, По-видимому, «наивный» метод ЭП для теории множеств просто расходится в присутствии аксиомы бесконечности.

\section{ЛИТЕРАТУРА}

1. Гильберт Д., Бернайс П. Основания математики. 2. Теория доказательств. М., Мир, 1980.

2. Ackermann, W. // Math. Ann., 1940, 117, H 2, 162-194.

3. Kreisel, G. // J. Symbol. Log., 1951, 16, 241-267.

4. Parikh, R. J. // Trans. Amer. Math. Soc., 1973, 177, 29-36.

5. Гавриленко Ю. В. // ДАН СССР, 1984, 276, № 1, 18-22.

6. Божич E. C. // Вестн. МГУ. Мат., мех., 1985, 5, 37-41.

7. Коэн Дж. П. Теория множеств и континуум-гипотеза. М., Мир, 1969.

8. Ериов Ю. Л. // Вычислительные системы, 1986, 114, 3-10.

9. Минц Г. // Изв. АН ЭССР. Физ. Матем., 1981, 31, № 4, 376-382.

10. Справочная книга по математической логике. Т. 2. Теория множеств. М., Мир, 1984.

11. Takahashi, M. // Publs Res. Inst. Math. Sci., 1977, 12, 577-708.

12. Wang, $H$. A Survey of Mathematical Logic. Peking, Sci. Press; Amsterdam. North Holland, 1963.

Ннститут кибернетики

Академии наук Эстонской ССР
Поступила в редакцию

$18 /$ I 1988

\section{G. MINTS}

\section{EPSILON-ASENDUSTE MEETOD PÄRANDLIKULT LÖPLIKE HULKADE TEOORIAS}

On esitatud epsilon-asenduste meetodi püstitus ja koonduvuse tōestus pärandlikult lõplike hulkade teoorias. 


\section{G. MINTŜ \\ EPSILON-SUBSTITUTION METHOD FOR THE THEORY OF HEREDITARY FINITE SETS}

The epsilon-substitution method introduced by Hilbert was later used for extracting numerical content of arithmetic proofs, and in particular for estimating the complexity of the resulting program. Essential features of the method are the applicability to classical (not necessarily intuitionistic) derivations and independence of their propositional structure: the complexity of the extracted program (and the rate of convergence of the method) depends only on the nesting of bound variables. So it is desirable to extend this method from the first-order arithmetic to other theories. We treat the theory of hereditarily finite sets in detail, and show how such extension can be made for several of its formalizations. The main technical result of this paper is the convergence of the epsilon-substitution method for the theory of hereditarily finite sets. It is established by the method given in a previous paper by the author (Proc. Acad. Sci. ESSR. Phys. Math., 1982, 31, № 4). It also extends the Ackermann theorem on the convergence of the epsilon-substitution method for the first-order arithmetic. 